\title{
The Vertical Mean Wind Profile Over the Ocean for Light to Moderate Winds
}

\author{
KENNETH W. RUGGLES ${ }^{1}$ \\ Dept. of Meteorology, Massachusetts Institute of Technology, Cambridge \\ (Manuscript received 22 October 1969, in revised form 20 February 1970)
}

\begin{abstract}
Analysis of 299 wind profile observations collected at the Massachusetts Institute of Technology oceanographic research platform provides a detailed picture of the behavior of wind profile parameters. A plot of roughness length vs velocity suggests the existence of classical hydrodynamic phenomena such as Jeffreys' minimum wind speed and the Kelvin-Helmholtz instability. The friction velocity appears to be, in general, a linear function of the wind velocity, except in regions where discontinuities exist. It is suggested that the air flow regime over the ocean in the wind range of $2-10 \mathrm{~m} \mathrm{sec}^{-1}$ (measured at a height of $10 \mathrm{~m}$ ) is best described as a region of constant drag coefficient $\left(C_{D}=1.6 \times 10^{-3}\right)$ modified by the superposition of almost singular departures at a number of discrete wind speeds, apparently caused by the onset of distinct instabilities at the water's surface.
\end{abstract}

\section{General experiment}

The nature of the vertical mean wind profile over the ocean remains one of the central issues in the study of the atmospheric boundary layer over the ocean. Consequently, an experimental study has been undertaken in an effort to increase our knowledge of processes in this regime through observation. Over a period of two years, measurements of mean wind speeds at vertically separated stations have been collected by Seesholtz (1968) and this author. This effort has resulted in a data set of 299 mean wind profiles measured in the first $10 \mathrm{~m}$ of the atmosphere. The profiles have a distribution as a function of the wind speed at $10 \mathrm{~m}$, taken as the reference height wind speed throughout this report, as shown in Fig, 1. The results of the study are valid in the lightto-moderate wind speed regime.

Each profile is based on wind speeds measured simultaneously from 4-8 anemometer stations. The lowest station was within $1 \mathrm{~m}$ of the mean sea surface unless sea conditions required otherwise, while the uppermost station varied in height between 8 and $12 \mathrm{~m}$. While instrument configurations varied, in every case the anemometer stations were positioned more or less logarithmically, with instruments concentrated within the first few meters above the water's surface. Since the instruments were attached to a rigid spar standing in $60 \mathrm{ft}$ of water, the measurements are free of effects such as buoy or ship motion while the behavior of the wind waves can be expected to be that of deep-water wind waves. All wind speeds have been averaged over 10-min intervals. The nearest upwind obstacle in the direction of the prevailing wind was Block Island, a distance of $26 \mathrm{mi}$. All fetches were thus $26 \mathrm{mi}$ or better.

\footnotetext{
${ }^{1}$ Current affiliation: Navy Weather Research Facility, Suit-
} land, Md.

\section{Analysis}

The basis for the analysis and discussion is the logarithmic wind profile, defined as

$$
U(z)=\frac{U_{*}}{\kappa} \ln \left(\frac{z}{z_{0}}\right)
$$

where $z_{0}$ is the roughness length, defined at that height at which $U(z)$, the mean wind at height $z$, is equal to zero. This parameter is normally thought of as characterizing the effect of the boundary on the mean air flow. The other parameters are the friction velocity $U_{*}$, and von Kármán's constant $\kappa$, taken to be equal to 0.42 . From an experimental viewpoint, $U_{*}$ and $z_{0}$ can be

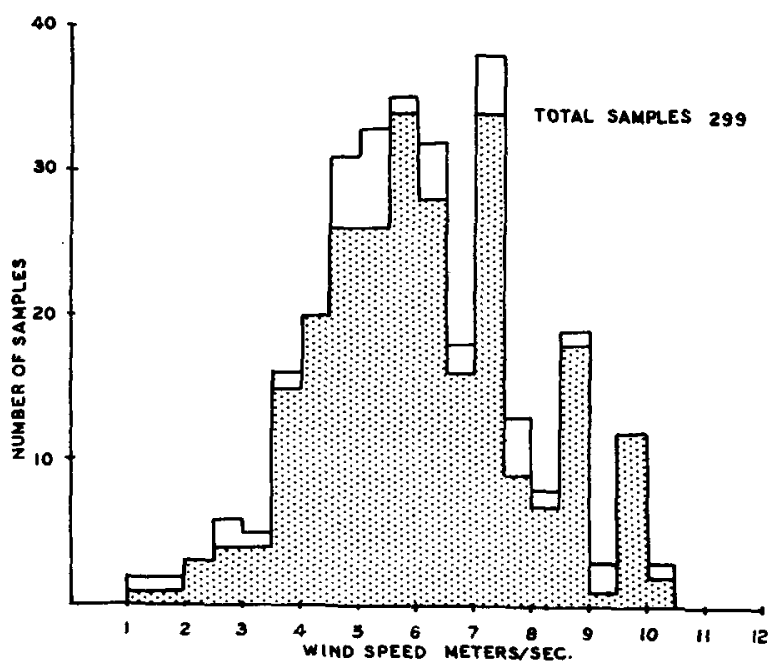

FIG. 1. Distribution of wind profile samples. Shaded area is distribution of profiles having a correlation with a $\log$ profile of 0.94 or better. 
TABLE 1. Distribution of correlation coefficient between $U_{*}$ and $z_{0}$.

\begin{tabular}{cc}
\hline Correlation coefficient & Number of samples \\
\hline 1.00 & 86 \\
$0.980-1.00$ & 106 \\
$0.950-0.980$ & 59 \\
$0.920-0.950$ & 26 \\
$0.890-0.920$ & 11 \\
$0.860-0.890$ & 2 \\
$0.830-0.860$ & 3 \\
$0.800-0.830$ & 2 \\
$0.740-0.800$ & 0 \\
$0.700-0.740$ & 4 \\
\hline
\end{tabular}

uniquely determined from (1), provided the profile is, in fact, logarithmic. These parameters become indeterminate, however, for experimental data representing non-logarithmic profiles unless one has a prior knowledge of the functional form of the profile. Therefore, experimental profiles which are not logarithmic have been discarded. Non-logarithmic profiles can result from a number of causes, such as stability changes, a passing ship upwind of the observation station, etc. Aside from the indeterminacy problem, discarding anomalous profiles excludes extraneous information which might otherwise bias the results and their subsequent interpretation. Fortunately, while valid cases of non-logarithmic profiles exist over the ocean, they are the anomalies. In this study, $13 \%$ of the data sample has been discarded. Of that $13 \%$, more than half of the cases were discarded on valid physical grounds. Therefore, better than $90 \%$ of the valid measured profiles were Iogarithmic, as shown in the shaded areas of Fig. 1.

Since (1) is linear in $\ln z$, it can be written as

$$
\ln z=\frac{\kappa}{U_{*}} U(z)+\ln z_{0},
$$

and one can use linear regression techniques to find the best least-squares fit of the assumed profile form to the observed data. Following Hoel (1954, p. 127), (2) can be written in terms of the observed wind speed $V$ at instrument height $Y_{i}$ in the form

$\ln z \ln Y_{i}$

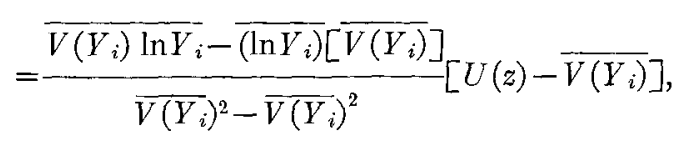

where the bar represents an ensemble mean which is defined in the usual sense for discrete data as

$$
\bar{f}=\frac{1}{N} \sum_{i=1}^{N} f_{i}
$$

$N$ being the number of profile points measured for each profile. From (3) the equation of the line best fitting the profile is determined. From this line, $U_{*}$ and $z_{0}$ can be directly computed, along with a linear correlation coefficient

$$
r=\frac{\overline{V\left(Y_{i}\right) \ln Y_{i}}-\overline{\left(\ln Y_{i}\right)}\left[\overline{V\left(Y_{i}\right)}\right]}{\left[\overline{\left.\left.\left(\overline{\ln Y_{i}^{2}}\right)-\left(\overline{\ln Y_{i}}\right)^{2}\right]^{1}\left[\overline{V^{2}\left(Y_{i}\right)}-\overline{V\left(Y_{i}\right.}\right)^{2}\right]^{1}}\right.},
$$

which measures how well the logarithmic profile form represents the observed profile.

\section{Results}

Table 1 lists the distribution of correlation coefficients computed from each of the observed mean wind profiles. A correlation coefficient of 0.94 has been chosen as the division point between those profiles defined as logarithmic and those discarded as deviating from the logarithmic form. The shaded region of Fig. 1 represents the distribution of the logarithmic wind profiles after the anomalous profiles had been discarded. Note that those profiles taken to be logarithmic are distributed as a function of the $10 \mathrm{~m}$ wind speed roughly the same as the distribution of the total sample. Each of the discarded profiles were individually examined, with the following results:

1) Of the four profiles with correlation coefficients $<0.74$, three were measured during a tine period when the mean wind speed suddenly increased at the upper levels during the averaging period. The fourth profile could not be classified due to insufficient supplementary information.

2) The profiles with correlation coefficients between 0.94 and 0.80 consist of 19 profiles measured during experiments with artificial sea slicks. These are not considered representative of a true marine environment. The remaining cases, though not attributed to any identifiable cause, were characterized by intermittency. That is to say, for a set of consecutive profiles there would be one or two anomalous profiles dispersed among an otherwise fully logarithmic set.

After the profiles were computed and sorted, those profiles defined as logarithmic were grouped into classes according to the $10 \mathrm{~m}$ wind speed. While the data were computed for a number of class intervals, an interval of $50 \mathrm{~cm} \mathrm{sec}^{-1}$ was chosen as optimum for displaying the data. A broader class interval retains the gross features, but tends to smear the detail. Narrower class intervals result in a discontinuous histogram. The class boundaries fall on the integral wind speed values and on the $0.5 \mathrm{~m} \mathrm{sec}^{-1}$ values of reference wind speed. An observed wind speed which fell exactly on the boundary was grouped with the higher adjacent class. The data in each class were used to compute a mean friction velocity and roughness length appropriate for the class.

The roughness length as a function of reference mean wind speed is shown in Fig. 2. The structure of this parameter was quite unanticipated. The first appearance of this structure resulted in extensive recomputation, 
including the shifting of the class intervals by one-half an interval width; however, the features persisted. Wu (1969) recently published a paper with data processed in somewhat similar fashion. While Wu did not take note of it, his roughness length plot shows peaks similar to those in Fig. 2 at 4 and $8 \mathrm{~m} \mathrm{sec}^{-1}$. Wu's graphs were drawn without any data discrimination and for wider class intervals. Therefore, the peaks did not stand out as markedly as they do in Fig. 2. Additional corroborating evidence of an indirect kind is provided by Kinsman's (1968) review of the Beaufort wind scale, shown in Fig. 5. Note that the natural wind speed division points selected on the basis of the appearance of the sea surface to shipborne observers coincides closely with the peaks in the roughness length graph.

On the basis that marked changes in roughness length characterize changes in the condition of the underlying surface as sensed by the atmosphere, one can speculate on the significance of each of the peaks. If an air stream were to be introduced over a perfectly calm sea, and then its velocity slowly increased, there should exist some velocity at which the first wind waves will appear. Below this velocity the atmosphere should be expected to sense a smooth aerodynamic boundary. Above this velocity, the atmosphere should feel a boundary with different aerodynamic characteristics. In Fig. 2 such a change occurs at $2 \mathrm{~m} \mathrm{sec}^{-1}$, suggesting this as the minimum wave-generating wind speed. This velocity is equivalent to a $1 \mathrm{~m}$ wind speed of $1.5 \mathrm{~m} \mathrm{sec}^{-1}$, and is in close agreement with Jeffreys' minimum wind speed for wind generation as discussed in Lamb (1932, p. 625).

The roughness length peak at $4 \mathrm{~m} \mathrm{sec}^{-1}$ cannot be explained. To the knowledge of the author, there isn't any theoretical work which could be used as a basis for explaining this peak. A tentative physical explanation will be suggested later, based on an analysis of the velocity defect graph of Fig. 5. The marked peak at 8.5 $\mathrm{m}_{\text {. }} \mathrm{sec}^{-1}$, however, is reminiscent of the widely discussed

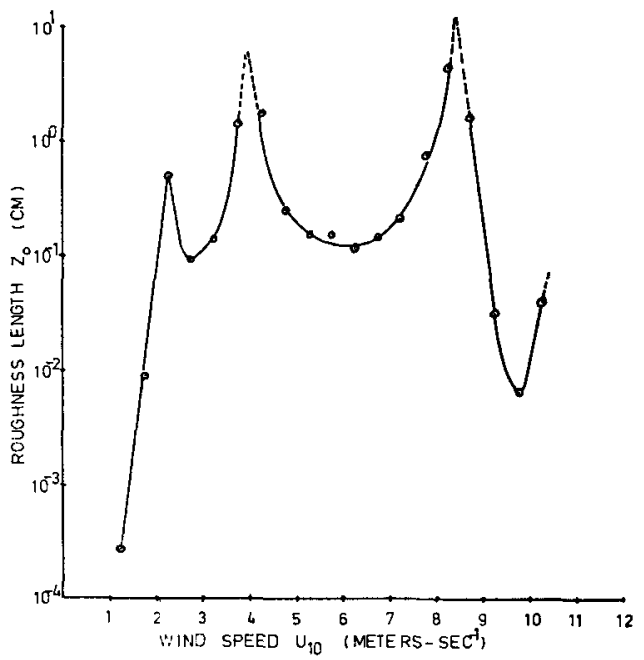

FIG. 2. Roughness length as a function of wind velocity.

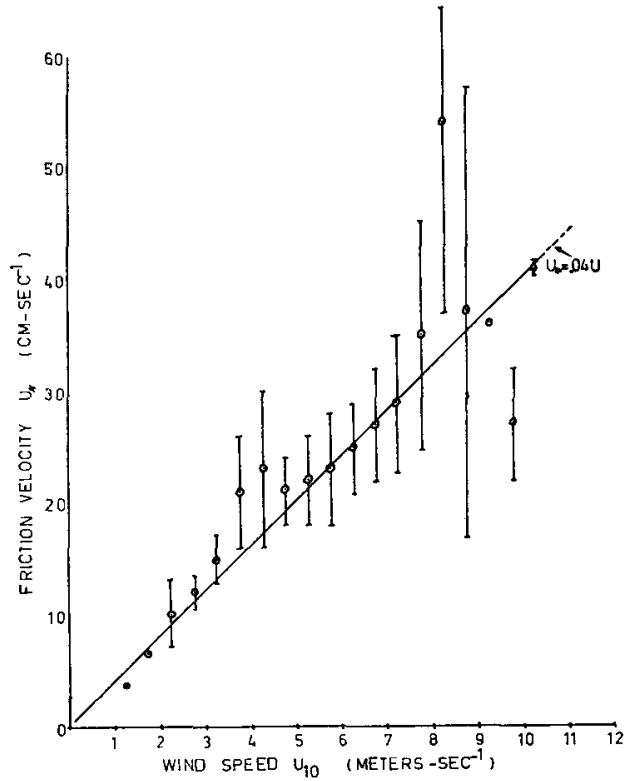

FIG. 3. Friction velocity as a function of wind velocity.

Kelvin-Helmholtz shear instability, predicted by Munk (1947) to occur at $6.5 \mathrm{~m} \mathrm{sec}^{-1}$. The higher observed wind velocity for the onset of the shear instability is attributed to the modification of the surface tension of the sea water, due to dissolved sea salts and contaminants.

Friction velocity as a function of reference wind speed (Fig. 3) shows a strong linear dependence upon velocity. For the $0-10 \mathrm{~m} \mathrm{sec}^{-1}$ speed range, the friction velocity can be approximated by

$$
U_{*}=0.04 U(10),
$$

where $U(10)$ is the $10 \mathrm{~m}$ wind speed. Note that the friction velocity plot shows only minor deviations from its trend in the vicinity of 2 and $4 \mathrm{~m} \mathrm{sec}^{-1}$. At $8.5 \mathrm{~m} \mathrm{sec}^{-1}$, however, the friction velocity appears to be strongly influenced by the same processes which affect the roughness length parameter. This strong effect is again what one might expect in the case of the KelvinHelmholtz instability, where the boundary instability changes the character of the entire turbulent regime.

The drag coefficient at height $z$ is defined as

$$
C_{D}(z)=\left[\frac{U_{*}}{U(z)}\right]^{2},
$$

its observed variation with wind speed being displayed in Fig. 4. Again, the singularities are clearly evident, as is the constant drag coefficient of $1.6 \times 10^{-3}$ between singularities. The deviation bars in Fig. 4 are also appropriate for the roughness length graph of Fig. 2, although deviation bars were not shown there due to the nonlinear scale of the graph. Therefore, the behavior of the drag coefficient in the vicinity of the singularities is also typical of the behavior of the values of the friction 


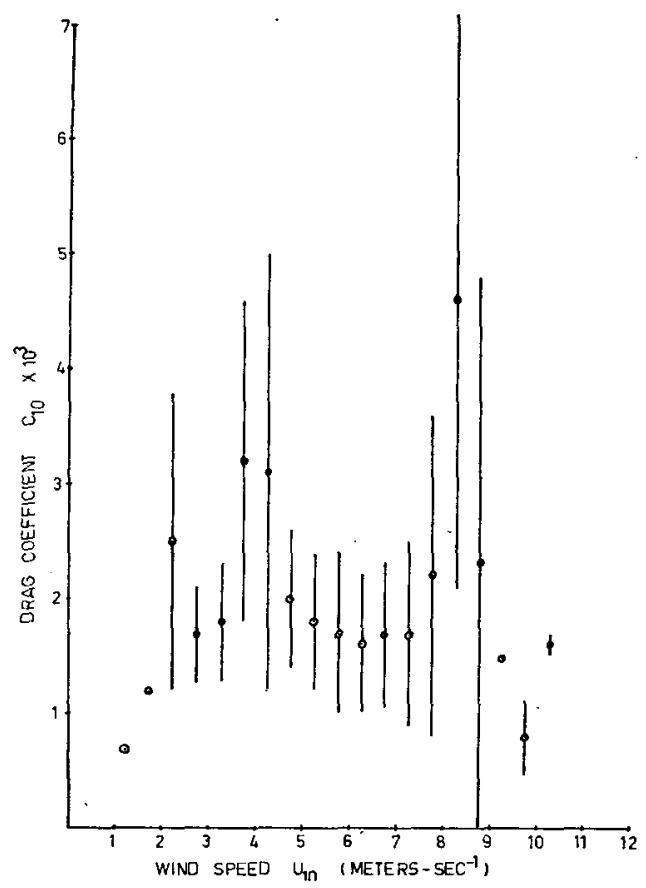

FIG. 4. Drag coefficient as a function of wind velocity.

velocity. This wide deviation at the singularities suggests that the variables have a modal structure within the class interval. This is what one would expect for sharp discontinuities. Such a structure would result in a mean for the class interval lying somewhere between the two modes of the variable, and extremely large and somewhat meaningless variance values. The plausibility of the modal structure has been investigated by examining the distribution of the drag coefficient within a class interval centered about the discontinuities as compared with a similarly chosen class in a velocity region removed from the discontinuities. In Table 2, the distribution of the drag coefficient within a class interval centered about the discontinuities is listed and compared with a

TABLE 2. Distribution of drag coefficients within selected $1 \mathrm{~m} \mathrm{sec}^{-1}$ class intervals.

\begin{tabular}{|c|c|c|c|}
\hline \multirow[b]{2}{*}{$\begin{array}{c}\text { Drag } \\
\text { coefficient } \\
C_{D}\left(\times 10^{3}\right)\end{array}$} & \multicolumn{3}{|c|}{ Wind speed intervals $\left(\mathrm{m} \mathrm{sec}^{-1}\right)$} \\
\hline & $\begin{array}{c}\bar{U}=3.5-4.5 \\
\left(4.0 \mathrm{~m} \mathrm{sec}^{-1}\right. \\
\text { discontinuity) }\end{array}$ & $\begin{array}{c}\bar{U}=8.0-9.0 \\
(8.5 \mathrm{~m} \mathrm{sec}-1 \\
\text { discontinuity) }\end{array}$ & $\begin{array}{c}\bar{U}=6.0-7.0 \\
\text { (no discontinuity) }\end{array}$ \\
\hline $0.5-1.0$ & 0 & 11 & 2 \\
\hline $1.0-1.5$ & 1 & 5 & 21 \\
\hline $1.5-2.0$ & 9 & 1 & 17 \\
\hline $2.0-2.5$ & 8 & 0 & 3 \\
\hline $2.5-3.0$ & 2 & 0 & 2 \\
\hline $3.0-3.5$ & 3 & 1 & 1 \\
\hline $3.5-4.0$ & 2 & 0 & 0 \\
\hline $4.0-4.5$ & 1 & 1 & 0 \\
\hline $4.5-5.0$ & 0 & 0 & 0 \\
\hline $5.0-5.5$ & 5 & 1 & 0 \\
\hline $5.5-6.0$ & 2 & 5 & 0 \\
\hline $6.0-6.5$ & 0 & 0 & 0 \\
\hline$>6.5$ & 2 & 2 & 0 \\
\hline Total cases & 35 & 27 & 46 \\
\hline
\end{tabular}

randomly chosen class removed from the discontinuities. Note the clearly evident bimodal structure of the drag coefficient for those classes centered about the discontinuities, while the $\bar{U}=6.0-7.0 \mathrm{~m} \mathrm{sec}^{-1}$ class has a rather uniform distribution.

The functional dependence of the drag coefficient on wind velocity has been a matter of extensive debate, based on scattered data. The results presented here suggest that the inferred functional dependence of the drag coefficient would depend more on how fast the wind was blowing during the various investigators' measurements or how the data were grouped, than on any physical relationship. The value of $1.6 \times 10^{-3}$ for the drag coefficient agrees with laboratory values of the drag coefficient determined by eddy correlation techniques, as summarized by Roll (1965, p. 158), and the values measured in the field using eddy-correlation techniques reported by Weiler and Burling (1967).

The computed mean values of $U_{*}$ and $z_{0}$ for each class interval were used in Eq. (1) to compute a mean $10 \mathrm{~m}$ wind speed for each velocity class. It was expected that the computed speed would closely approximate the specified mean wind speed for the class. In most cases, however, this computation resulted in a consistent underestimate of the wind speed appropriate for the class. The magnitude of this underestimate is shown in Fig. 5. Since we have been processing the data using statistical approaches, this result should be interpreted from a statistical viewpoint. The implicit assumption in

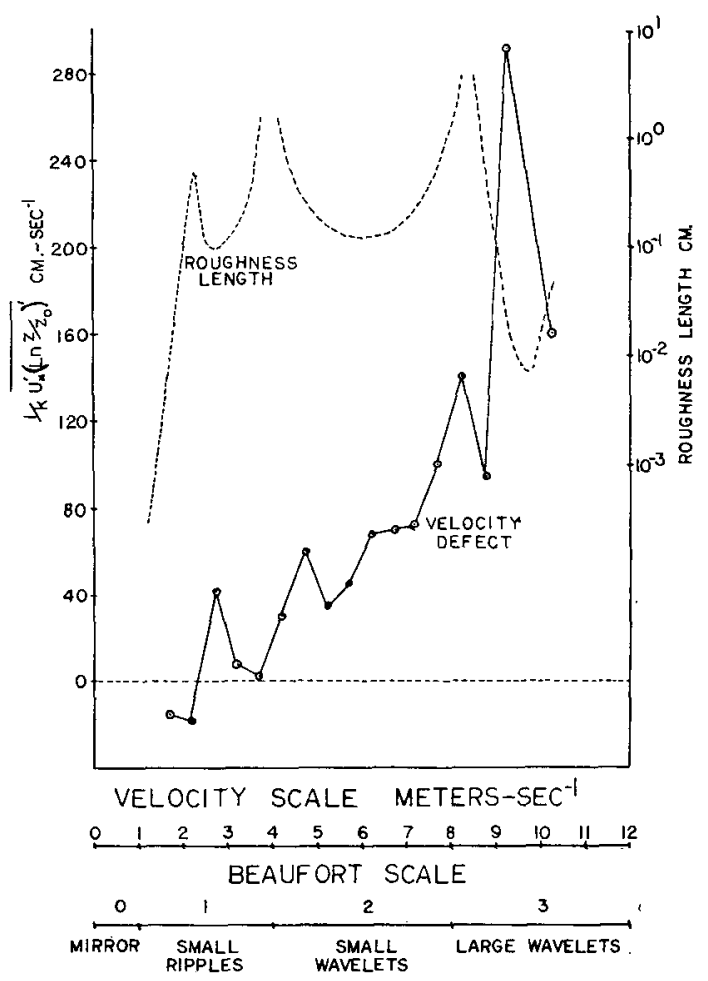

FIG. 5. Roughness length and velocity defect (see text) as a function of wind velocity and Beaufort wind scale. 
(1) is that $U_{*}$ and $z_{0}$ are each independent parameters required to describe the flow. If, however, they are not statistically independent, the mean profile form can be expected to deviate from that inferred from (1) by the amount of correlation between $U_{*}$ and $z_{0}$. We will thus represent $U_{*}$ and $z_{0}$ as the sum of mean quantity and a deviation from the mean, i.e.,

$$
\begin{gathered}
U_{*}=\overline{U_{*}}+U_{*}{ }^{\prime}, \\
\ln \left(\frac{z}{z_{0}}\right)=\left[\overline{\left.\ln \left(\frac{z}{z_{0}}\right)\right]+\left[\ln \left(\frac{z}{z_{0}}\right)\right]^{\prime}}\right\} .
\end{gathered}
$$

After substituting (7) into (1) and taking an ensemble mean, we have

$$
\left.\bar{U}(z)=\frac{1}{K} \bar{U} * \overline{\left[\ln \left(\frac{z}{z_{0}}\right)\right.}\right]+\overline{\frac{1}{K} U *^{\prime}\left[\ln \left(\frac{z}{z_{0}}\right)\right]^{\prime}} .
$$

Note that this is not the same type of averaging process as is done in the familiar Reynolds convention. In this case we are averaging over an ensemble defined by all those values of $U_{*}$ and $z_{0}$ which fall within a specified class interval, rather than over an arbitrary time period. Eq. (8) indicates that for any given class of wind profile data, the mean wind speed at a height $z$ can be represented as the sum of a mean profile component and a term representing the correlation between $U_{*}$ and $z_{0}$.

An effort to establish causes for the behavior of the correlation terms shown in Fig. 5 first suggested analysis of deviations in the assumed profile form due to atmospheric stability. The Monin-Obukhov log-linear profile will be used to examine this aspect. Following Lumley and Panofsky (1964, p. 107), we write

$$
U(z)=\frac{U_{*}}{\kappa}\left[\ln \left(\frac{z}{z_{0}}\right)+\frac{\beta z}{L^{\prime}}\right],
$$

where

$$
L^{\prime}=\frac{U_{*} T}{{ }_{\kappa}}\left(\frac{\delta \bar{U}}{\delta z}\right) /\left(\frac{\delta \theta}{\delta z}\right),
$$

$L^{\prime}$ being the "modified" Monin-Obukhov length scale. The modified length scale is chosen as more appropriate for use with the profile-derived stress. The other parameters are $\beta$, an imperical constant; $g$, the acceleration due to gravity; and $\theta$, the potential temperature.

Rewriting (9) in terms of $\mathrm{Ri}$, the Richardson number, where

$$
\mathrm{Ri}=\frac{g}{T}\left(\frac{\delta \theta}{\delta z}\right) /\left(\frac{\delta U}{\delta z}\right)^{2}
$$

we have

$$
U(z)=\frac{U_{*}}{\kappa}\left[\ln \left(\frac{z}{z_{0}}\right)+\beta \operatorname{Ri}\left(\frac{\delta U}{\delta z}\right) z / U_{*}\right] .
$$

Differentiating Eq. (1) and substituting the result into (11), we have

$$
U(z)=\frac{U_{*}}{\kappa}\left[\ln \left(\frac{z}{z_{0}}\right)+\frac{\beta \mathrm{Ri}}{\kappa^{2}}\right] .
$$

This last step is justified on the basis that the $U_{*}$, as used in the analysis, is derived from, and therefore directly related to, Eq. (1). We now subject (12) to the same analysis used to derive (8), with the result that

$$
\begin{aligned}
& U(z)=\frac{1}{\kappa} \overline{U_{*}} \overline{\left[\ln \left(\frac{z}{z_{0}}\right)\right]}+\frac{1}{\kappa} \overline{U_{*}{ }^{\prime}\left[\ln \left(\frac{z}{z_{0}}\right)\right]^{\prime}} \\
& +\frac{\overline{U_{*}}}{\kappa} \beta^{\prime} \mathrm{Ri},
\end{aligned}
$$

where $\beta^{\prime}=\beta / \kappa^{2}$.

Subtracting (1) from (13), we now show that

$$
\overline{U_{*}^{\prime}\left[\ln \left(\frac{z}{z_{0}}\right)\right]^{\prime}}=-\overline{U_{*} \beta^{\prime}} \mathrm{Ri} .
$$

Two conclusions can be drawn from Eq. (14):

1) For increasing values of $\mathrm{Ri}$ (i.e., increasing stability), we can expect an increasing correlation between $U_{*}$ and $\left[\ln \left(z / z_{0}\right)\right]$, but in a negative sense.

2) Noting from Eq. (5) the relationship between $U_{*}$ and $U$, we can also expect an increasing correlation between $U_{*}$ and $\left[\ln \left(z / z_{0}\right)\right]$ for increasing wind velocity, but again in a negative sense.

Reexamining Fig. 5, it is immediately apparent that the observed effect is exactly opposite from that which would be expected from stability on the basis of conclusion 2). Furthermore, reasoning that the increased vertical mixing expected at higher wind velocities would tend to reduce stability effects, the observed effects do not agree with the expected effect on the basis of conclusion 1). Therefore, the phenomena displayed in Fig. 5 must be due to causes other than stability effects on the profile analysis.

We thus look for other alternatives: for wind flow over a rigid boundary, where the nature of the surface is independent of the flow parameters, the correlation term would, in theory, go to zero and (8) would revert to the familiar logarithmic profile. We can interpret the first term on the right-hand side of (8) as representing the mean velocity at any height required to support atmospheric dynamics, while the second term identifies profile modification due to external effects. In view of the data screening, the effects of errors in determining the profile descriptors should be expected to be random, rather than systematic from class to class in the fashion shown in Fig. 5. Therefore, the second term on the right-hand side of (8) is taken to represent the wind at any height required to support the interaction of the boundary with the wind field. For simplicity, we will write Eq. 
(8) as

$$
U=U_{p}+U_{w}
$$

where $U_{p}$ is the profile component of the mean wind, and $U_{w}$ the boundary interaction component of the mean wind.

While $U_{p}$ is always a positive quantity, as shown in Fig. 5, $U_{w}$ takes on both positive and negative values, with a transition at about $2 \mathrm{~m} \mathrm{sec}^{-1}$. Given the mean parameters of $\bar{z}_{0}$ and $\bar{U}_{*}$, the momentum $U_{p}$ at any level $z$ is all that is required to effect a balance between the momentum created at some higher level and the momentum transferred or lost due to friction. The additional momentum, $U_{w}$, represents either the excess or deficit of momentum, where the positive sign indicates the excess, at level $z$ needed to support wave growth.

Looking at (15) in another way, if the ocean did not transfer momentum with the atmosphere, only $U_{p}$ would be present. The ability of the vertical mean wind profile to sustain its logarithmic character suggests that the atmospheric source or sink of transferred momentum does not reside soley in the near-ocean boundary layer, but in some higher level. It is speculated that $U_{w}$ is a measured momentum passing through level $z$ to support boundary processes.

Fig. 5 indicates that at wind speeds $>2 \mathrm{~m} \mathrm{sec}^{-1}$ in the mean there is an excess of momentum at level $z$ available for wave generation and maintenance. At wind speeds $<2 \mathrm{~m} \mathrm{sec}^{-1}$ in the mean the atmosphere does not appear to have adequate momentum to support frictional losses, and may receive momentum from the ocean, presumably from the underlying swell. This interpretation is consistent with the view that there is a minimum wave-generation wind speed at $\sim 2 \mathrm{~m} \mathrm{sec}^{-1}$. Above $4 \mathrm{~m} \mathrm{sec}^{-1}$ the ratio of $U_{w} / U$ varies between 0.1 and 0.2. This ratio is consistent with the ratio of wavegrowth momentum to the total atmospheric momentum as determined by Stewart (1961).

\section{Instrument errors and averaging time}

Discussion of several loose ends has been postponed until this point, since they are more logically viewed in retrospect. Let us now ask what effect instrument errors and averaging time may have on the results.

The implicit assumption in the choice of a mean windaveraging time is that the processes we study are stationary over the averaging interval. In studying the atmosphere over the ocean, the air-sea boundary can change so rapidly that stationarity arguments become highly suspect, even for short averaging intervals. On the other hand, if the averaging time is too short, the profile data will reflect the effect of individual waves or wave groups rather than the effect of the mean wind field. Seesholtz investigated this averaging time problem as related to the use of wind profile data to predict windwave growth. He concluded that short averaging intervals were a necessary condition for realistic wave- growth estimates. At the same time his data show that a 10-min averaging time appears to be the minimum one can use and still get consistent profiles. It appears from the Seesholtz results that a 10 -min mean wind is near optimum, and thus has been used in this study.

Considering instrument accuracy, McCready (1966) demonstrated that under certain conditions large errors can be introduced into mean wind speed measurements. Bernstein (1967) discusses the effect of some of these errors on wind profile measurements. All of the measurements reported herein were made using fast-response cup anemometers with distance constants $<1 \mathrm{~m}$. The cups used in the experiments were selected from a number of identical anemometers on the basis of matched wind response characteristics. The anemometers were mounted at various heights at various times in an attempt to avoid biases introduced by a consistent error in a single station. The $w$ directional response of the cups was measured and found to be consistent with that reported by McCready. For observations made by the author, a wind vane was mounted at the $8 \mathrm{~m}$ station, and a continuous wind direction record was maintained. The standard deviation $\sigma_{\theta}$ of the wind direction never exceeded $10^{\circ}$, while under $5^{\circ}$ was common. The wind was blowing over a far more uniform field than that commonly encountered in experiments over land. While there probably were small instrument errors, the consistency of the results argues in favor of their small magnitude. Independent simultaneous measurements of $-\overline{U^{\prime} W^{\prime}}$ by eddy correlation techniques in four out of five cases agreed within $10 \%$ of the profile value of $U_{*}{ }^{2}$. The fifth case is believed to be a special case, occurring at the $8 \mathrm{~m} \mathrm{sec}^{-1}$ discontinuity. Finally, the profiles grouped into each of the class intervals were measured under a variety of stability and wind-wave generating conditions. Some of the deviation of the observed values of $U_{*}$ and $z_{0}$ certainly can be attributed to anemometer errors; however, the deviation bars do not appear to reduce systematically with increased wind speed, as one would expect if instrument errors, as discussed by McCready and Bernstein, were the dominant contribution to the measured variability.

\section{Critical appraisal}

This study is essentially an analysis of a group of carefully measured wind profiles, using two separate data sets collected by two investigators in two different years using different instrument arrays. Both data sets, taken independently or together, provide the same general results. It is the author's feeling that the parameters presented accurately represent the observed conditions. The key question is, "What were the observed conditions?" Unfortunately, this study cannot answer this question. The momentum lost by the atmosphere can be roughly partitioned in to that required to generate wind waves, that required to maintain existing waves, and that lost to other effects such as friction and drift 
currents. Failure to consider this momentum partition during the observations leaves unanswered the key question as to whether these plots are characteristic of a generating sea, a falling sea, or a fully-arisen sea. For example, in examining the modal structure in the vicinity of the discontinuities, are the modes characteristic of two physical states, such as wave-generating conditions as contrasted with non-generating conditions, or are the modes the result of unique values of the variables characteristic of a sub-class within the chosen class interval? One can only conclude that the plots represent some "typical" observed mean profile state. Consequently, their use in applications such as windwave generation prediction schemes may be ill-advised.

In retrospect, the value of identifying sea state and sea state trend with each measured vertical mean profile becomes obvious. It would appear that the next step would be the sorting of all profile data into three categories: 1) profiles measured over a generating sea, 2) profiles measured over a fully arisen sea, and 3) profiles measured over a falling sea.

Each of these profile samples could be individually analyzed using techniques similar to those discussed herein, providing the answers to questions about what the profiles and the correlation terms in Fig. 5 represent.

\section{Conclusions}

Charnock (1955) suggested that $z_{0}$ should be proportional to $U_{*}^{2} / g$, resulting in the wind profile formula

$$
U(z)=\frac{U_{*}}{\kappa} \ln \left[\beta z /\left(\frac{U_{*}}{g}\right)^{2}\right],
$$

where $\beta$ is a constant of proportionality. Inspection of Fig. 2 clearly shows that $z_{0}$ does not vary as $U_{*}{ }^{2}$. Laboratory experiments by $\mathrm{Wu}$ (1968) resulted in similar findings; however, Wu noted that the Charnock expression is appropriate at high wind speeds. This expression implicity assumes that a single surface roughness parameter will specify the momentum balance requirements of both the ocean and the atmosphere. There is no reason for this to be so. It is suggested that a more appropriate approach is the separation of the profile into two components, one resulting from atmospheric dynamics and one from wind-wave interaction, as in Eq. (15).

At low wind speeds, the vertical mean wind profile parameters are all functions of velocity, with the roughness length parameter being highly structured. Two of the three peaks in the roughness length graph can be explained by recourse to classical hydrodynamics. The peak at $4 \mathrm{~m} \mathrm{sec}^{-1}$ requires explanation. The rise and then the return to a near zero value of the velocity defect in Fig. 5 at $4 \mathrm{~m} \mathrm{sec}^{-1}$, taken along with the roughness length plot, suggests to this author that the peak at $4 \mathrm{~m} \mathrm{sec}^{-1}$ may be associated with a transition from capillary waves to gravity waves as being those waves which have a dominant effect on the atmosphere. The evidence is far too meager and the argument far too tenuous, however, to make this assertion with any firm conviction.

Acknoreledgments. The assistance and encouragement of Prof. Erik Mollo-Christensen is gratefully acknowledged. This project is supported by the Office of Naval Research under Contract Nonr N00014-67-A-0204-0024, and by the National Science Foundation under Grant 1439.

\section{REFERENCES}

Bernstein, A. B., 1967 : A note on the use of cup anemometers in wind profile experiments. $J$. Appl. Meteor., 6, 280-286.

Charnock, H., 1955: Wind stress on a water surface. Quart. J. Roy. Meteor. Soc., 81, 639-640.

Hoel, P. G., 1954: Introduction to Mathematical Statistics. New York, Wiley, $331 \mathrm{pp}$.

Kinsman, B., 1968: An exploration of the origin and persistance of the Beaufort wind force scale. Tech. Rept. 39, Chesapeake Bay Institute, The Johns Hopkins University.

Lamb, H., 1932: Hydrodynamics. Reprint by Dover Publ., New York.

Lumley, J. L., and H. A. Panofsky, 1964: The Structure of Atmospheric Turbulence. New York, Interscience, $239 \mathrm{pp}$.

McCready, P. B., Jr., 1966: Mean wind speed measurements in turbulence. J. Appl. Meteor., 5, 219-225.

Munk, W., 1947: A critical wind speed for air-sea boundary processes. J. Marine Res., 6, 203-218.

Roll, H. U., 1965: Physics of the Marine Atmosphere. New York, Academic Press, 426 pp.

Seesholtz, J. R., 1968: A field investigation of air flow immediately above ocean surface waves. Tech. Rept., Massachusetts Institute of Technology, Dept. of Meteorology.

Stewart, R. W., 1961 : The wave drag of wind over water. J. Fluid Mech., 10, 189-194.

Weiler, H. S., and R. W. Burling, 1967 : Direct measurements of stress and spectra of turbulence in the boundary layer over the sea. J. Atmos. Sci., 24, 653-664.

Wu, J., 1968; Laboratory studies of wind-wave interactions. $J$. Fluid Mech., 34, 91-122.

- 1969: Wind stress and surface roughness at air-sea interface. J. Geophys. Res., 74, 444-455. 